Richard Wilkinson / Kate Pickett

The spirit level: Why more equal societies

almost always do better

London, Allen Lane, 2009

ISBN 978-1-84614-039-6

\title{
Fabiola Cabrera*
}

Investigadora Economía

Biblioteca del Congreso Nacional

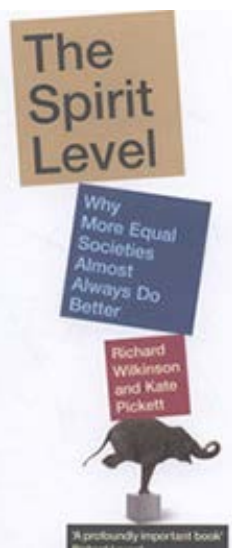

económico sin precedentes, con disminuciones sustanciales en los niveles de pobreza, y un importante aumento generalizado en el ingreso de las personas, sin embargo, este éxito económico no se ha visto acompañado con la misma fuerza en las variables sociales.

Cuando se visualiza gráficamente la relación entre el ingreso per cápita y la esperanza de vida (figura 1), se observa que en los países de ingresos bajos, la esperanza de vida es sustancialmente mayor en la medida que aumenta el ingreso, sin embargo, esta relación no se ajusta a una relación lineal para niveles superiores y aparentemente aumentar los niveles de ingreso no tiene impacto en la cantidad de años que las personas logran vivir. Si este mismo ejercicio, se

*fcabrera@bcn.cl 
repite para observar la relación entre el ingreso y los niveles de felicidad (figura 2), se observa que en niveles de ingreso inferiores a los 10 mil dólares per cápita, el ingreso no es relevante, luego entre 10 y 25 mil aproximadamente, un mayor ingreso se condice con mayores niveles de felicidad pero para los países de ingresos altos, por sobre los 25 mil dólares per cápita, un ingreso más alto pareciera no tener relación con mayores niveles de felicidad. Esta es la hipótesis que Wilkinson y Pickett sostienen durante su libro: que una vez alcanzados ciertos niveles de vida en donde la subsistencia se tiene relativamente resuelta, el factor que repercute fuertemente en la sociedad no es el ingreso sino la desigualdad. Dejando muy en claro que este trabajo no pretende trazar una relación causa-efecto se sostiene que los países más igualitarios tienen un "mejor vivir para todos". 


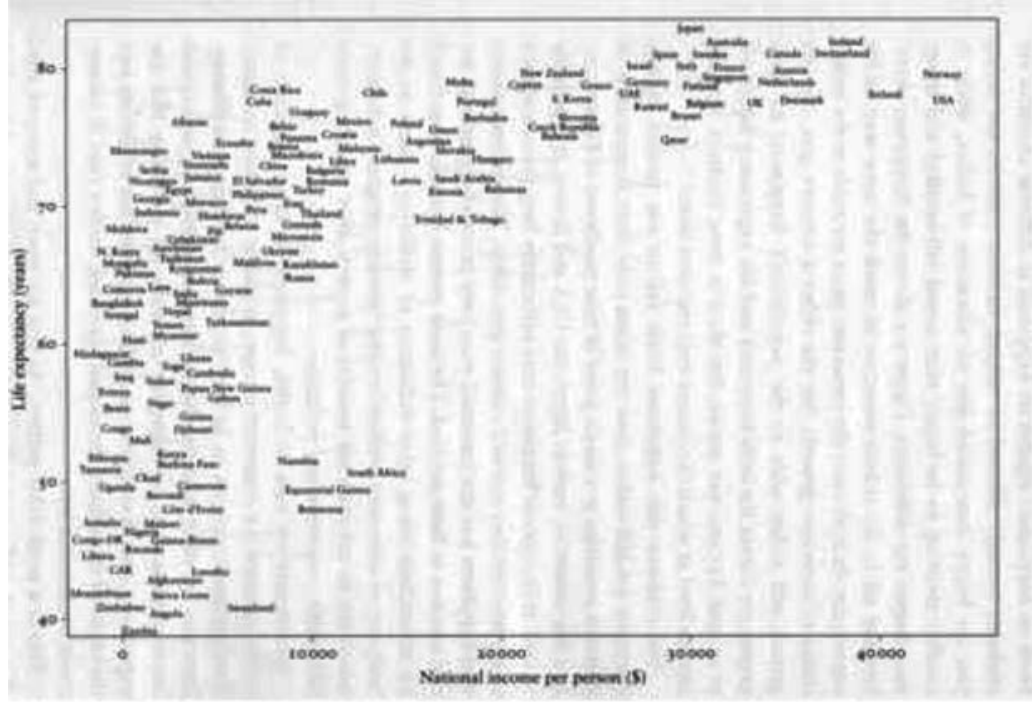

Figura 1. Eeectativa de Vida con reladión al ingreso nadional per cipita. Fuente Wilineon y Pidkett. 2009

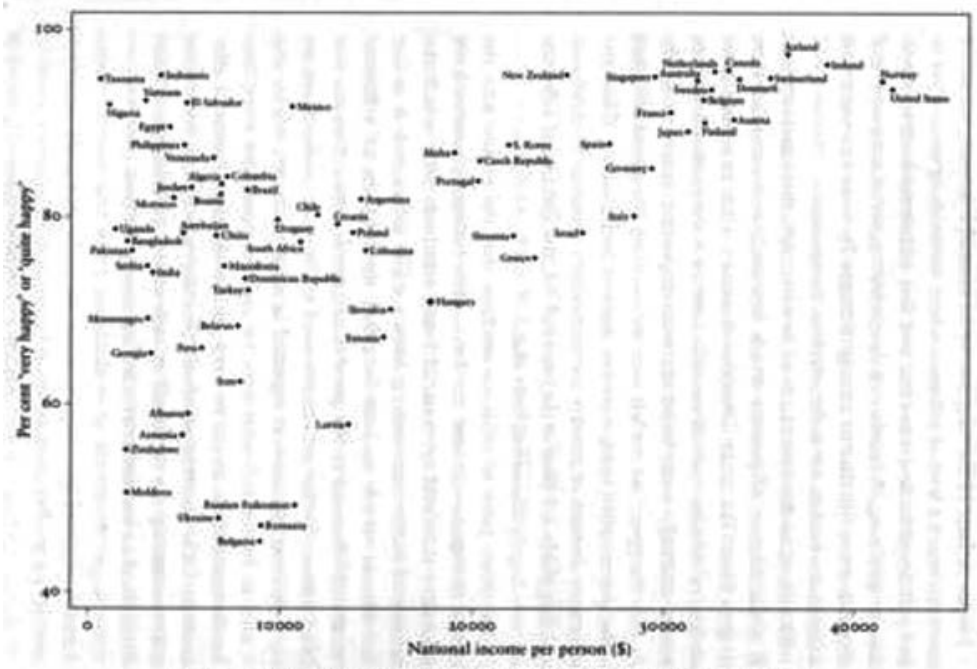

Figure 1.2 Happiness and avenase incomes (data for UK anaruilable).'

Figura 2: Feliodad e ingreso Promedio (Poino Unido no disponible), Fuente Wikincon y Pidkett. 2009

En el libro se revisa las correlación entre la distribución del 
ingreso para países considerados de ingresos altos y un amplio set de variables sociales que incluyeron: la salud física, salud mental, abuso de drogas, educación, tasas de encarcelamiento, obesidad, movilidad social, confianza y vida comunitaria, violencia, embarazos en la adolescencia y bienestar de los niños. En orden de mejor a peor equidad según el ratio 20/20 los países son los siguientes: Japón, Finlandia, Noruega, Suecia, Dinamarca, Bélgica, Austria, Alemania, Países Bajos, España, Francia, Canadá, Suiza, Irlanda, Grecia, Italia, Israel, Nueva Zelanda, Australia, Reino Unido, Portugal, EE.UU. y Singapur.

Los detractores de establecer una relación entre desigualdad y diversos indicadores sociales, argumentan que los indicadores sólo reflejan el promedio de las condiciones individuales y que en un país con alta inequidad los malos indicadores sociales como por ejemplo en salud, desempeño en educación y otros ámbitos, se explicaría porque las personas más adineradas gozarían de mejores condiciones que aquellos con menos recursos y por ende, sólo mejorando las condiciones de estos últimos debería mejorar el promedio de los indicadores sociales del país. Esta idea reforzaría la hipótesis que los indicadores están más correlacionados con los niveles de ingresos ya que las desigualdades atañen directamente al individuo, mientras que la desigualdad es una característica de un sistema social. Sin embargo, cuando se observa el extenso material que los autores analizan, en donde a su vez se ven las condiciones sociales entre estratos sociales en comparación con los otros países, dicha hipótesis pierde fuerza.

En el ámbito de la salud, mientras que en países de bajos niveles de ingreso, tienen importancia las enfermedades infecciosas como la tuberculosis, el cólera o el sarampión, en países con mayores ingresos las causas de muerte se relacionan con enfermedades derivadas del estrés, obesidad o del corazón. Dentro de los países con mayores ingresos, estas afecciones muestran peores indicadores en los países con una mayor desigualdad pero no solo en ellas sino también con otro tipo de enfermedades que aparentemente no tienen ninguna relación, tal es el caso de las enfermedades pulmonares o cáncer. El 
ejemplo más robusto mencionado, es la comparación entre Suecia y Estados Unidos, y entre Suecia e Inglaterra y Gales en donde se muestran que variables como las tasas de mortalidad y mortalidad infantil, enfermedades como cáncer, diabetes, hipertensión, entre otras, no sólo son más comunes dentro de grupos sociales más bajos en los países más desiguales, sino también en los grupos de mayores ingresos en comparación con los mismos grupos en países más equitativos. Esto implicaría que en los países más desiguales no sólo a los más pobres sufren con peor salud, sino la condición trasciende a toda la sociedad. Este es el argumento más fuerte que advierte sobre la necesidad de una mejor distribución. Aun cuando alguien pertenezca al $1 \%$ más rico de un país, su propensión a ciertas enfermedades será mayor que aquella que afecta al 1\% de los países más equitativos. Una mejor equidad no solo se traduce en una mejora en la calidad de vida en los grupos socio-económicos menos favorecidos sino también favorece a los grupos de mayores ingresos.

En lo que respecta a la obesidad, este es efectivamente un problema de países más ricos, ya que no es la falta de alimentos sino el exceso de ellos. Lo paradójico en esto es que la obesidad afecta a las personas de estratos sociales más pobres. Del grupo de países revisados, EE.UU. ocupa por lejos el liderazgo en mayores tasas de obesidad, tanto general como infantil. Sin duda la comida rápida, que representa un menor costo que la comida saludable, tiene un rol en estas tasas, sin embargo tiene relación con muchas otras variables como el estilo de vida, educación de los padres, hábitos deportivos, entre otros. La relación de esto con la distribución del ingreso puede ser explicada con la asociación entre la insatisfacción y la comida. Se citan varios estudios donde se encuentra un vínculo entre situaciones de estrés e ingesta de azúcares y grasas, lo que aplica para personas y animales. La búsqueda de rápida satisfacción y felicidad en escenarios con alta desigualdad apoyaría la hipótesis que a mayor desigualdad mayor obesidad. Si así fuere, la obesidad parece ser una trampa para la clases sociales más bajas, en la cual a mayores inequidades existe una mayor insatisfacción y por ende mayor la probabilidad de ingerir alimentos en busca de satisfacción. 
En otro ámbito, la relación entre las disparidades de ingreso y de embarazos adolescentes es quizá la menos intuitiva. Algunas hipótesis sostienen una relación entre un medio ambiente estresante que fomentaría una reacción biológica temprana de madurez sexual y consecuentemente, los embarazos en adolescentes. Con el embarazo, las madres jóvenes pasan rápidamente a pertenecer a un grupo de adultos que las hace abandonar, su condición adolescente, o al menos sentir que lo hacen y con ello acceder a toda una red social constituida por madres y padres del entorno social.

De manera similar a la salud, la educación pertenece al individuo y por lo tanto, podría decirse que sus niveles de ingresos determinarán su rendimiento en la educación. Sin embargo, la relación entre desigualdad y educación proporciona varias dimensiones de investigación, la educación de los padres, los ambientes sociales y todos los elementos que afectan en el proceso de aprendizaje son potenciales variables. Hay varios factores implícitos en la desigualdad que afectan directamente en el rendimiento estudiantil, en primer lugar, las situaciones de estrés tienen consecuencias psicológicas que pueden impedir a los individuos tener un proceso cognitivo normal. Experimentos psicológicos demostraron que los niños de clase baja tienen un peor rendimiento que los de clases más acomodadas sólo cuando se evidencian las diferencias sociales. Así lo demostraron Hoff y Pandey (2004) al constatar en nińos de la India que el rendimiento entre las clases sociales se diferenció sólo cuando sus castas fueron reveladas. Por otra parte, estudiantes de secundaria Afro-americanos tuvieron peores resultados que estudiantes blancos, sólo después de anunciar que están siendo testeados. Este último resultado fue calificado como "la amenaza del estereotipo" (Steele y Aronson, 1995). Adicionalmente, Siddiqi (2007) destaca que la puntuación de Pisa es más alta en los países con una larga historia de amplios regímenes de Estados de bienestar o Sistemas Sociales Públicos, por el contrario los países con menos prestaciones sociales reciben las peores puntuaciones.

Con relación a la movilidad social, las disparidad en la distribución del ingreso es una variable observada en un momento 
determinado del tiempo, si existe una desigualdad alta o baja puede no ser tan significativa como la dinámica dentro de este cuadro. Así, si en un determinado período de tiempo las personas en el decil más bajo se encuentran posteriormente en una clase superior y viceversa, la distribución del ingreso cobra menos relevancia, al contrario, si las clases sociales tienden a estar estancadas a través del tiempo, implica que la gente en grupos sociales más bajos no tienen ninguna posibilidad de ascender en la escala social y en este caso, la inequidad cobra mayor fuerza porque entonces se aborda un tema de justicia social.

Los estudios en este campo son metodológicamente difíciles, en general el estatus social de los padres deben ser comparados con el de su descendencia, por lo que es necesario hacer un seguimiento de cohortes a través de generaciones lo que requiere al menos 25 años de investigación. Cuando no puede llevarse a cabo este complejo proceso, una metodología alternativa es el porcentaje de remuneraciones explicada por la remuneración de los padres, indicador utilizado en este trabajo. De acuerdo a ello, los países más equitativos registran una mayor movilidad social.

La relevancia de esta variable también se explica porque da cuenta de una dinámica de persistencia y acumulación generándose círculos virtuosos para las clases sociales más adineradas y círculos viciosos para los estratos menos favorecidos. El sentido común indica que las personas de mayor ingreso, disponen de más recursos con los cuales pueden acceder a más y mejores oportunidades que les permitirá ampliar las oportunidades a su descendencia, contrariamente la situación que viven quienes poseen menos.

La idea de la persistencia es apoyada con varias teorías, por una parte, existe un grupo de razones en el marco de la transmisión familiar que dificultan la movilidad social intergeneracional, esta transmisión se da con la riqueza, la transmisión de las capacidades y la transmisión de los gustos y preferencias. Por otra parte, los mercados de capitales son imperfectos existiendo discriminación hacia quienes tienen menos, razón por la cual personas emprendedoras pero con menos recursos no pueden acceder a créditos que les permita 
invertir para acceder a una situación más favorable. Un tercer aspecto dice relación con las segregaciones, situación que se observa en vecindarios que tienden a reproducir la situación económica del lugar hacia quienes lo habitan y en último lugar existen las inequidades autocumplidas en dos formas: la primera de ellas se relaciona con la discriminación y las profecías auto cumplidas de individuos que, teniendo posibilidades para lograr un ascenso en la escala social, el grupo de pertenencia los lleva a verse a sí mismos encajados en un grupo del cual no se puede escapar, y la segunda, se refiere al discurso dominante, que plantea que los grupos con mayores ingresos mantienen un discurso sobre la prevalencia de las clases sociales que desincentiva a las personas en clases de menos ingresos tomar iniciativas que puedan generar movilidad social. Además de la persistencia, la acumulación refuerza las desigualdades en el tiempo, ya que la transmisión de la riqueza o nivel educacional dentro de los hogares puede ser analizado como un activo que impacta en los ingresos futuros para los hijos.

Wilkinson y Pickett recopilan una gran cantidad de valioso material para cada una de las variables revisadas en el libro, y quizá uno de los aportes más trascendentales, es la comprensión de que de alguna u otra manera todas ellas se encuentran en una relación de interdependencia. No requiere mucho esfuerzo comprender cómo la violencia, el uso de drogas, las tasas de homicidio y los niveles de reclusión son más altas en sociedades muy desiguales. Las carencias relativas pueden conducir a la frustración y la hostilidad y con ello dar paso a un encadenamiento de privaciones y refuerzos de las dinámicas persistentes.

Como Wilkinson y Pickett sugieren, la relación entre los indicadores sociales y la distribución del ingreso es un análisis complejo de múltiples variables y fuerzas interconectadas que interactúan al mismo tiempo. El sentido común indica que son comprensibles dadas las dinámicas de autorefuerzo. De esta manera, países más igualitarios tienen mayores niveles de confianza, menos violencia, mejor desempeño escolar, menos embarazos adolescentes, menos población carcelaria, una alta movilidad social, mejor 
esperanza de vida, mayor bienestar infantil, mejor salud mental y física y menor consumo de drogas y niveles de obesidad, entregando un marco de análisis coherente que permite visualizar la inequidad como un fenómeno que requiere de una perspectiva más amplia. La figura 3 permite visualizar lo anterior al mostrar la relación entre un indicador compuesto de variables sociales y la inequidad.

\section{Health and social problems are worse in more unequal countries}

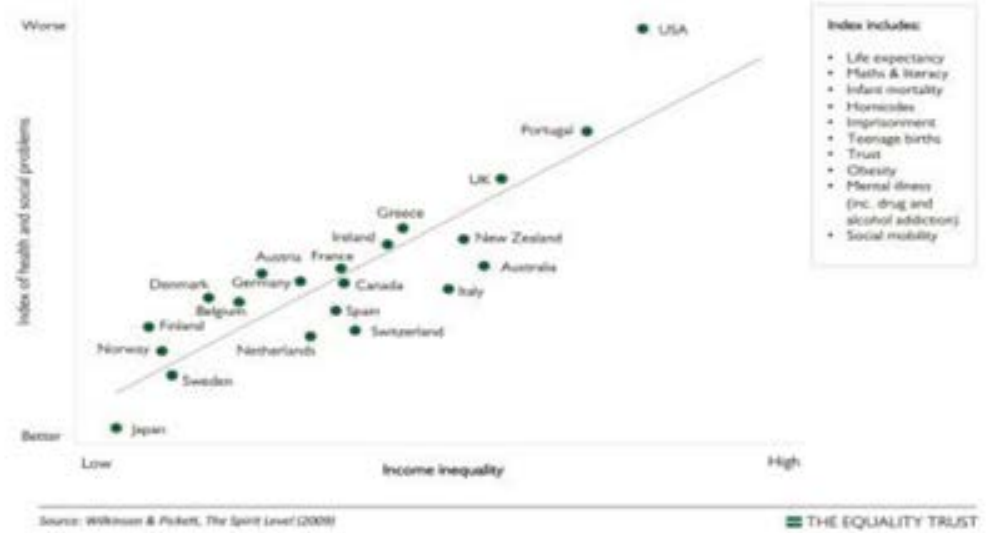

Elaboración propia

Latinoamérica concentra las desigualdades más altas del mundo y Chile no es la excepción, aun cuando su modelo económico es reconocido como exitoso, la inequidad ha sido persistente y es una reconocida barrera para el desarrollo. Las reformas liberales implementadas a partir de mediados de la década de los setenta, establecieron las bases que propiciaron el crecimiento económico pero la dimensión social quedó postergada. Posteriormente, durante los gobiernos democráticos se generó una notable reducción de la pobreza del 44,5\% en 1990 al 14,4\% en el 2013, sin embargo, los altos niveles de desigualdad prevalecen hasta ahora. Revisando las mismas variables en The Spirit Level para el caso chileno, se observa el mismo patrón encontrado en los otros países seleccionados por los autores, es decir, si Chile se incorporara en el trabajo de Wilkinson y 
Pickett, considerando que posee una peor distribución que el grupo seleccionado, Chile registraría en general los peores indicadores del grupo.

Actualmente, las desigualdades son motivo de preocupación también para países desarrollados, lo que lleva a cuestionarse qué hay detrás de este fenómeno y qué posibles alternativas son viables teniendo en cuenta la complejidad que involucra. La inequidad está en aumento, en octubre de 2008 la OCDE publicó el reporte "Crecimiento Desigual?" dando cuenta que la brecha entre ricos y pobres ha crecido en más de tres cuartas partes de los países de la OCDE y que el crecimiento económico de las últimas décadas ha beneficiado más a los ricos de los pobres. Similar diagnóstico tuvo Thomas Piketty en su libro el Capital en el S XXI y otros autores en diversas publicaciones como Joseph Stiglitz y Ha Joon Chang por solo mencionar algunos.

En este marco, la reciente investigación del PNUD sobre la desigualdad en Chile publicada recientemente en Desiguales, de los investigadores Matías Cocińa, Raimundo Frei y Osvaldo Larrańaga, resulta también un material fundamental para la comprensión de un fenómeno, que como sociedad, nos afecta a todos. 